\title{
Author index Volume 4
}

Abo-Elsoud, M. A., Experimental and computational simulation studies on creep deformation mechanisms of a novel nanostructured $\mathrm{Cu}$ and $\mathrm{Cu}-10 \% \mathrm{Sn}$ Alloy

2 (2015) 1550008

Abu-Shams, M., see Shabib, I.

4 (2015) 1550022

Adlakha, N., see Kumari, B.

1 (2015) 1550002

Ameri, M., see Varshney, D.

3 (2015) 1550015

Ashrafi, H. \& Shariyat, M., A mathematical boundary integral equation for analysis of the heterogeneous media, using the functionally graded elements

3 (2015) 1550017

Azari, A., Bahraini, A. \& Marhamati, S., A CFD technique to investigate the chocked flow and heat transfer characteristic in a micro-channel heat sink

Bahraini, A., see Azari, A.

2 (2015) 1550007

2 (2015) 1550007

Bhattacharya, S., Pamnani, G., Sanyal, S. \& Sharma, K., Numerical simulation of crack propagation under fatigue loading in piezoelectric material using extended finite element method

4 (2015) 1550025

Bulaeva, E. A., see Pankratov, E. L.

1 (2015) 1550006

Cao, L., see Li, S.

1 (2015) 1550003

4 (2015) 1550024

Gao, R. \& Li, Y., Theoretical study on the electronic structure, mechanical property, and thermal expansion of yttrium oxysulfide

$1(2015) 1550004$

Guo, S.-S., see Li, Y.

1 (2015) 1550001

Guo, X., see Qiu, J.

3 (2015) 1550013

Hassan, A., Hossain, MD. F., Rana, MD. S. \& Kouzani, A. Z., Theoretical study of quantum capacitance and associated delay in armchair-edge graphene nanoribbons

3 (2015) 1550019

He, Y., see Li, Y.

1 (2015) 1550001

Hossain, MD. F., see Hassan, A.

3 (2015) 1550019

Hussain, F., see Khanday, M. A.

2 (2015) 1550012

Iyakutti, K., see Rajarajeswari, M.

3 (2015) 1550014 
Jayakumar, K., see Kalpana, P.

3 (2015) 1550018

Joseph, J. \& Lu, Y. C., Effect of graphene layer thickness on effective modulus of 3D CNT/Graphene nanostructures

$2(2015) 1550010$

Kalpana, P., Jayakumar, K. \& Nithiananthi, P., Effect of geometry on the pressure induced donor binding energy in semiconductor nanostructures

3 (2015) 1550018

Kaur, R. P., Sawhney, R. S. \& Engles, D., Proliferating conduction by isomerism

4 (2015) 1550024

Kawazoe, Y., see Rajarajeswari, M.

3 (2015) 1550014

4 (2015) 1550020

Khan, M. R., see Shabib, I.

4 (2015) 1550022

Khanday, M. A., Hussain, F. \& Nazir, K., Tissue necrosis and passage of fluid due to cold stress from the thermally damaged human body peripherals: A mathematical model

2 (2015) 1550012

3 (2015) 1550019

Kouzani, A. Z., see Hassan, A.

Krasnyuk, I. B., Surface-directed multi-dimensional wave structures in confined binary mixtures

$4(2015) 1550023$

Krishna, S. A. M., Shridhar, T. N. \& Krishnamurthy, L., Computational investigation on thermal conductivity behavior of $\mathrm{Al}$ 6061-SiC-Gr hybrid metal matrix composites

4 (2015) 1550021

Krishnamurthy, L., see Krishna, S. A. M.

4 (2015) 1550021

Krishnamurthy, L., see Mohan Krishna, S. A.

3 (2015) 1550016

Kumari, B. \& Adlakha, N., Two-dimensional finite difference model to study temperature distribution in SST regions of human limbs immediately after physical exercise in cold climate

1 (2015) 1550002

Lakshmi, I., see Rajarajeswari, M.

3 (2015) 1550014

2 (2015) 1550011

1 (2015) 1550003

Li, D., see Li, S.

Li, S., Li, D., Cao, L. \& Shangguan, Z., Parameter estimation approach for particle flow model of rockfill materials using response surface method

$1(2015) 1550003$

Li, Y., Guo, S.-S., He, Y. \& Liu, Z., A simplified constitutive model for predicting shape memory polymers deformation behavior

1 (2015) 1550001

Li, Y., see Gao, R.

1 (2015) 1550004

Liu, Z., see Li, Y.

1 (2015) 1550001

Lu, Y. C., see Joseph, J.

2 (2015) 1550010 
Mahesh, R., Rajagopalan, M. \& Palanivel, B., Doping effect on electronic band structure and magnetic properties of MFeAs $(\mathrm{M}=\mathrm{Li}, \mathrm{Na})$

$1(2015) 1550005$

Marhamati, S., see Azari, A.

2 (2015) 1550007

Mohan Krishna, S. A., Shridhar, T. N. \& Krishnamurthy, L., Computational investigation on thermal expansivity behavior of $\mathrm{Al} 6061-\mathrm{SiC}-\mathrm{Gr}$ hybrid metal matrix composites

Mousa, A. A. \& Khalifeh, J. M., Structural, electronic and elastic properties of the B2-ScM $(\mathrm{M}=\mathrm{Au}, \mathrm{Hg}$ and $\mathrm{Tl}$ ) intermetallic compounds: $A b$ initio calculations

Nithiananthi, P., see Kalpana, P.

Odhiambo, H. \& Othieno, H., Ab initio study of the structural, vibrational and thermal properties of $\mathrm{Ge}_{2} \mathrm{Sb}_{2} \mathrm{Te}_{5}$

2 (2015) 1550009

Othieno, H., see Odhiambo, $\mathrm{H}$.

2 (2015) 1550009

Palanivel, B., see Mahesh, R.

1 (2015) 1550005

Pamnani, G., see Bhattacharya, S.

Pankratov, E. L. \& Bulaeva, E. A., An analytical

4 (2015) 1550025 approach to model manufacturing a drift heterobipolar transistors: On approach to optimize technological process to increase integration rate

1 (2015) 1550006

Qiu, J., Yi, Y. B. \& Guo, X., Computational prediction of electrical and thermal conductivities of disklike particulate composites

3 (2015) 1550013

Rajagopalan, M., see Mahesh, R.

1 (2015) 1550005

Rajarajeswari, M., Iyakutti, K., Lakshmi, I., Rajeswarapalanichamy, R. \& Kawazoe, Y., Functionalized single-walled carbon nanotube $(5,0)$ as a carrier for isoniazid - A tuberculosis drug

3 (2015) 1550014

3 (2015) 1550014

Rajeswarapalanichamy, R., see Rajarajeswari, M.

3 (2015) 1550019

4 (2015) 1550025

3 (2015) 1550015

4 (2015) 1550024

Sawhney, R. S., see Kaur, R. P.

Shabib, I., Abu-Shams, M. \& Khan, M. R.,

Nanoindentation response of Fe-10\% Cr bi-crystal structures with $\Sigma 5\langle 001\rangle$ and $\Sigma 3\langle 110\rangle$ tilt boundaries:

An atomistic study

4 (2015) 1550022

Shangguan, Z., see Li, S.

1 (2015) 1550003

Shariyat, M., see Ashrafi, H.

3 (2015) 1550017 
Sharma, K., see Bhattacharya, S.

Shridhar, T. N., see Krishna, S. A. M.

Shridhar, T. N., see Mohan Krishna, S. A.

Shriya, S., see Varshney, D.

Tuncer, E. \& l'Abee, R., Numerical modeling of non-woven fiber mats: Their effective mechanical and electrical properties

Varshney, D., Shriya, S., Sapkale, R., Varshney, M. \& Ameri, M., Pressure induced stiffening, thermal softening of bulk modulus and brittle nature of mercury chalcogenides

Varshney, M., see Varshney, D.

Yi, Y. B., see Qiu, J.
3 (2015) 1550015

$4(2015) 1550025$

4 (2015) 1550021

3 (2015) 1550016

3 (2015) 1550015

$2(2015) 1550011$

3 (2015) 1550015

3 (2015) 1550013 